\title{
NEUMONÍA ASOCIADA A VENTILACIÓN MECÁNICA EN LA UNIDAD DE CUIDADOS INTENSIVOS PEDIÁTRICOS DE UN HOSPITAL TERCIARIO, 2015-2018
}

\author{
PNEUMONIA ASSOCIATED WITH MECHANICAL VENTILATION IN THE PEDIATRIC INTENSIVE CARE UNIT \\ OF A TERTIARY HOSPITAL, 2015-2018 \\ Liliana Cieza-Yamunaqué ${ }^{1, a}$, Edgar Coila-Paricahua ${ }^{1, a}$
}

\begin{abstract}
RESUMEN
Introducción: La neumonía asociada a ventilador (NAV) aumenta 2 a 3 veces el riesgo de mortalidad. Objetivo: Conocer características de NAV, determinar tasas de infección, gérmenes aislados, sensibilidad antibiótica y mortalidad. Métodos: Estudio descriptivo, retrospectivo en pacientes con NAV 2015-2018. Los datos se obtuvieron del programa Rikchary de una unidad de cuidados intensivos pediátricos. Resultados: Se registraron 60 casos de NAV, con tasas promedio anuales de 9.8 por 1000 días ventilación mecánica (VM). El 65\% fueron tardías diagnosticadas después de 5 días de VM. Los días de VM promedio fueron de 15 , reingresaron a VM el $25 \%$. Los gérmenes más frecuentes fueron Stenotrophomona maltophilia sensible $81 \%$ al cotrimoxazol y Pseudomona aureginosa sensible $80 \%$ al cefepime, $90 \%$ a amikacina y entre el $50 \%$ a $60 \%$ a los carbapenemens. La mortalidad fue del $15 \%$. Conclusión: La tasa anual promedio de NAV en el servicio evaluado se encuentra dentro de estándares internacionales, el tipo más frecuente la tardía fue más frecuente en menores de 6 meses y sexo masculino. La desnutrición, cardiopatía congénita, tiempo de ventilación mecánica, días de hospitalización y cirugías se encontraron en mayor proporción en pacientes con NAV. Los gram negativos fueron los más frecuentes y la mortalidad $15 \%$.
\end{abstract}

Palabras clave: Neumonía asociada al ventilador; Infección hospitalaria; Unidades de cuidado intensivo pediátrico. (fuente: DeCS BIREME)

\begin{abstract}
Introduction: Ventilator-associated pneumonia (VAP) increases the risk of mortality by 2 to 3 times. Objectives: To know characteristics of VAP, determining VAP infection rates, analyze related factors, isolated germs, antibiotic sensitivity and mortality. Methods: Descriptive, retrospective study in patients with VAP 2015-2018. The data was obtained from the Rikchary program of a pediatric intensive care unit, analyzed with SPSS 24.00. Results: There were 60 cases of VAP, with average annual rates of 9.8 per 1000 days of mechanical ventilation (MV). $65 \%$ of the VAPs were late diagnosed after 5 days of MV, the average MV days were $15,25 \%$ of the patients were re-admitted to MV. The average hospitalization was 31 days. The most frequent germs were Stenotrophomona maltophilia sensitive $81 \%$ to cotrimoxazol and Pseudomona aureginosa sensitive $80 \%$ to cefepime, $90 \%$ to amikacin and between $50 \%$ to $60 \%$ to carbapenemens. The mortality was $15 \%$. Conclusion: The average annual rate of VAP in the evaluated service is within international standards, the most frequent type is late, was more frequent in children under 6 months and male. Malnutrition, congenital heart disease, mechanical ventilation time, days of hospitalization and surgeries were found in a greater proportion in patients with VAP. The most frequent negative gram and $15 \%$ mortality.
\end{abstract}

Key words: Pneumonia; Ventilator-associated; Cross infection; Intensive care units; Pediatric. (source: MeSH NLM)

${ }^{1}$ Hospital Nacional Edgardo Rebagliati Martins-EsSalud, Lima-Perú.

a Médico Pediatra Intensivista de la Unidad de Cuidados Intensivos Pediátricos.

Citar como: Liliana Cieza-Yamunaqué, Edgar Coila-Paricahua. Neumonía asociada a ventilación mecánica en la unidad de cuidados intensivos pediátricos de un hospital terciario, 2015-2018. Rev. Fac. Med. Hum. Julio 2019; 19(3):19-26. DOI 10.25176/RFMH.v19i3.2167

Artículo publicado por la Revista de la Facultad de Medicina Humana de la Universidad Ricardo Palma. Es un artículo de acceso abierto, distribuído bajo los términos de la Licencia Creative Commons: Creative Commons Attribution 4.0 International, CC BY 4.0 (https://creativecommons.org/licenses/by/4.0/), que permite el uso no comercial, distribución y reproducción en cualquier medio, siempre que la obra original sea debidamente citada. Para uso comercial, por favor póngase en contacto con revista.medicina@urp.pe 


\section{INTRODUCCIÓN}

La ventilación mecánica (VM) es una intervención de soporte vital muy utilizada en pacientes críticos en unidades de cuidados intensivos, pero al ser una técnica invasiva genera riesgos entre los que se encuentra las complicaciones infecciosas como la neumonía asociada a ventilación mecánica (NAV), la cual se define como la neumonía intrahospitalaria que ocurre en los pacientes después de 48 horas de tener un dispositivo para asistir controlar la respiración (traqueostomía o intubación endotraqueal) y que no estaba presente ni en período de incubación en el momento del ingreso; o que es diagnosticada a las 72 horas siguientes a la extubación y retirada de la ventilación mecánica1,2,3.

La NAV es una de las infecciones intrahospitalarias más frecuentes en las unidades de cuidados intensivos pediátricos: los niños que la desarrollan tienen aumento de 2 a 3 veces el riesgo de mortalidad, prolongación de la estancia hospitalaria e incremento del consumo de antimicrobianos (50\%) con el consecuente aumento del riesgo de resistencia antimicrobiana ${ }^{3}$. La mortalidad atribuible a NAV es controvertida, debido a la vulnerabilidad de los pacientes en unidades críticas, estimándose entre 5-25\%. Las tasas de infección por NAV oscilan entre 8 a $12 / 1000$ días de VM ${ }^{3,4}$.

La NAV sigue siendo un problema importante para la salud pública. Se produce por distintos mecanismos, principalmente a través del tubo endotraqueal o traqueostomía, pero también por aspiración de secreciones colonizadas procedentes de la orofaringe, por vía hematógena a partir de focos de infección distantes o de la flora intestinal a través de translocación bacteriana y por contigüidad desde infecciones adyacentes a los pulmones ${ }^{4}$. El conocimiento de su patogenia, características epidemiológicas y factores de riesgo, nos permiten tomar medidas preventivas para disminuir los casos, sabiendo que la prevención de NAV es un objetivo nacional de seguridad del paciente, $y$ constituye un indicador de calidad de la atención ${ }^{4,5,6}$.

La NAV de inicio temprano ocurre durante los primeros cuatro días de ventilación mecánica y generalmente es causada por bacterias sensibles a los antibióticos. La NAV de inicio tardío se desarrolla cinco o más días después del inicio de la VM y es causada por patógenos resistentes a múltiples fármacos $(\mathrm{MDR})^{7}$. El diagnóstico temprano de la NAV y un tratamiento antibiótico adecuado puede reducir la aparición de organismos resistentes ${ }^{7,8}$.
Conocer nuestra realidad permitirá tomar las medidas preventivas correspondientes para lograr disminuir las tasas de infección y una atención de calidad para nuestros pacientes pediátricos críticos $^{9}$, por ello el propósito principal de este trabajo es conocer las características de la neumonía asociada a ventilador en nuestra Unidad de Cuidados intensivos pediátricos (UCIP) en el período 2015 al 2018, determinar las tasas de infección de NAV así como los gérmenes aislados, su sensibilidad y la mortalidad debido a NAV.

\section{MÉTODOS}

Se realizó un estudio descriptivo, retrospectivo, de los pacientes con diagnóstico de neumonía asociada a ventilador en la UCIP del Hospital Edgardo Rebagliati Martins (HNERM) entre enero-2015 y diciembre-2018. La unidad de cuidados intensivos pediátricos del HNERM, atiende a pacientes críticos de alta complejidad, transferidos de todo el país, cuenta con nueve camas para niños de un mes hasta antes de los 14 años de edad, reportándose 254, 282, 298 y 318 egresos por año (2015, 2016, 2017 y 2018 respectivamente).

La definición de NAV se tomó de la Norma técnica del Ministerio de Salud del Perú 2016 y la Norma de vigilancia de ESSALUD 2016 ${ }^{10,11}$. La tasa de infección de neumonía asociada a Ventilador se obtiene dividiendo los episodios de NAV entre los días de VM por 1000. La NAV temprana se denominó a la neumonía de menos de 5 días y la tardía más de 5 días ${ }^{2,12}$.

Los datos se obtuvieron del programa Rikchary, un programa informático diseñado para registrar datos de pacientes hospitalizados en el servicio de UCIP. Se elaboró una base de datos en Microsoft Excel 2010, y para el análisis se utilizó estadística descriptiva con IBM SPSS Statistics 24.00, siguiendo los principios éticos de investigación.

\section{RESULTADOS}

Se registraron 60 casos de neumonías asociadas a ventilador en el período 2015 al 2018, de un total 1152 egresos, las tasas anuales de NAV se muestran en la Figura 1, siendo la tasa total de estos 4 años de 9.8 casos por 1000 días de VM. 


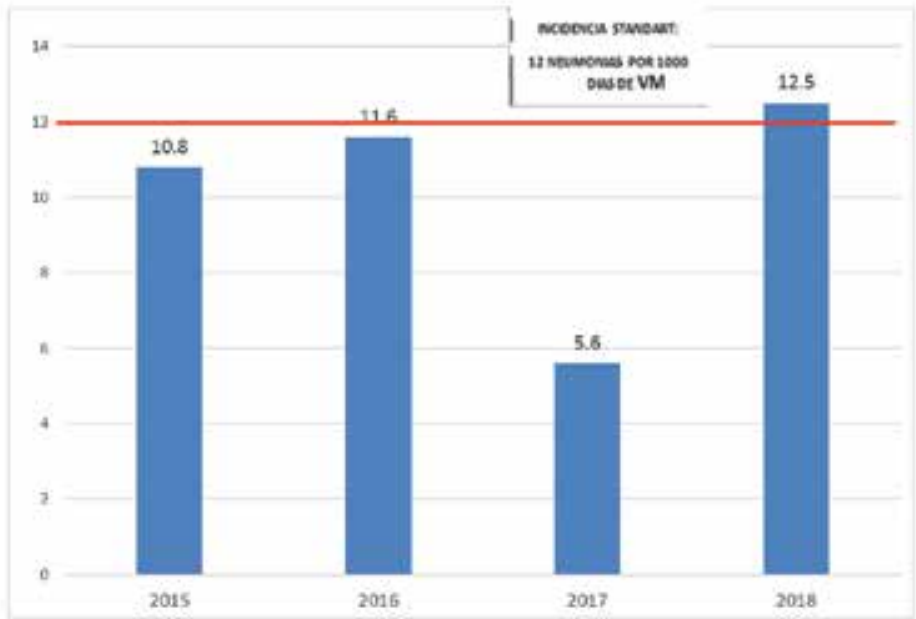

Figura 1. Tasa anual de neumonía asociada a ventilador (NAV) en la unidad de cuidados intensivos pediátricos del Hospital Rebagliati 2015-2018.

El número de episodios y el tiempo total de ventilación mecánica por año se observan en Tabla 1. Tres pacientes presentaron dos episodios de NAV durante su hospitalización en UCIP teniendo los tiempos de

hospitalización 38, 48 y 207 días cada uno. Un paciente presentó 3 episodios de NAV teniendo 142 días de hospitalización en UCIP.

Tabla 1. Número de casos por año de NAV y días de uso anual de ventilación mecánica en la unidad cuidados intensivos pediátricos del Hospital Rebagliati 2015-2018.

\begin{tabular}{lcccc} 
& $\mathbf{2 0 1 5}$ & $\mathbf{2 0 1 6}$ & $\mathbf{2 0 1 7}$ & $\mathbf{2 0 1 8}$ \\
\hline Egresos por año & 254 & 282 & 298 & 318 \\
$\mathrm{~N}^{\circ}$ de casos NAV* & 15 & 19 & 8 & 18 \\
Días de uso VM** & 1390 & 1644 & 1436 & 1440 \\
\hline
\end{tabular}

* NAV: Neumonia asociada a ventilador; ** VM : Ventilación mecánica.

La frecuencia de las NAV tempranas fue de 21 y tardía fue 39. La mediana de días de presentación de NAV es de 7 días en la totalidad de los casos, siendo 3 días para NAV temprana y 14 días para NAV tardía. El 53\% (32) fue del sexo masculino. El $38 \%$ de las NAV se presentaron en menores de 6 meses, pudiéndose observar en la Figura
2, frecuencia de NAV según grupo etáreo en relación con NAV temprana y Tardía y el porcentaje total según grupo etáreo, teniendo una mediana de edad de 15 meses (24 meses para NAV temprana y 10 meses para NAV tardía).

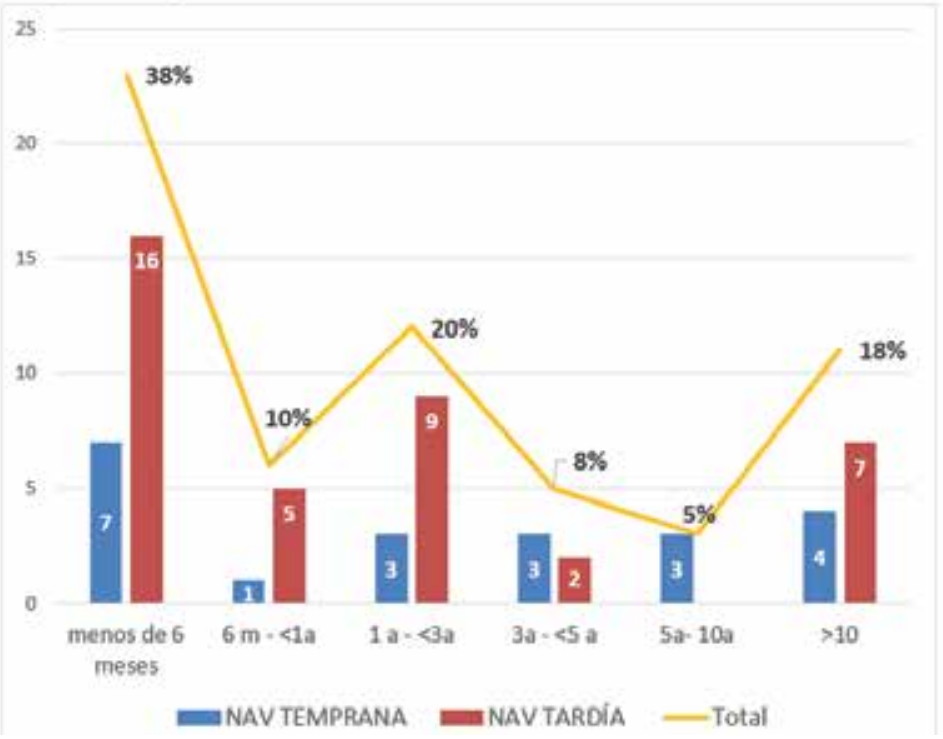

Figura 2. Frecuencia de la neumonía asociada a ventilador (NAV) y porcentaje según grupo etáreo en la unidad de cuidados intensivos pediátricos del Hospital Rebagliati 2015-2018. 
Las características de factores para NAV se frecuentemente comorbilidades cardíacas y indican en la Tabla 2. El $30 \%$ de los pacientes oncológicas; y en el rubro de otras se encuentran las con NAV eran desnutridos, $30 \%$ de los pacientes comorbilidades neurológicas (5\%) y respiratorias (5\%). eran post operados y el $57 \%$ de las NAV tenían

Tabla 2. Características de factores para con la neumonía asociada a ventilador (NAV) en la unidad de cuidados intensivos pediátricos del Hospital Rebagliati 2015-2018.

\begin{tabular}{|c|c|c|c|}
\hline & $\begin{array}{l}\text { NAV TEMPRANA } \\
(n=21)\end{array}$ & $\begin{array}{l}\text { NAV TARDÍA } \\
(n=39)\end{array}$ & $\begin{array}{l}\text { TOTAL } \\
(n=60)\end{array}$ \\
\hline \multicolumn{4}{|l|}{ Días de dispositivos } \\
\hline Días de VM*(mediana) & $11(3-142)$ & $15,5(3-88)$ & 15 \\
\hline Días CVC ${ }^{* *}$ ( mediana) & $12(5-40)$ & $14(6-43)$ & 13 \\
\hline Días sonda Foley( mediana) & $9(3-35 d)$ & $14(3-66 d)$ & $10,5 d$ \\
\hline \multicolumn{4}{|l|}{ Reingreso de VM } \\
\hline Si (frecuencia de pacientes) & $2(3 \%)$ & $13(22 \%)$ & $15(25 \%)$ \\
\hline \multicolumn{4}{|l|}{ Estado nutricional } \\
\hline Desnutrido & $6(10 \%)$ & $12(20 \%)$ & $18(30 \%)$ \\
\hline Eutrofico & $12(20 \%)$ & $22(37 \%)$ & $34(57 \%)$ \\
\hline Sobrepeso/obesidad & $3(5 \%)$ & $5(8 \%)$ & $8(13 \%)$ \\
\hline \multicolumn{4}{|l|}{ Días de hospitalización en UCIP } \\
\hline Promedio de días y rango & $19(8-142 d)$ & $38(8-207 d)$ & $31,5 d$ \\
\hline \multicolumn{4}{|l|}{ Post operados } \\
\hline $\mathrm{Si}$ & $10(17 \%)$ & $8(13 \%)$ & $18(30 \%)$ \\
\hline \multicolumn{4}{|l|}{ Cirugía } \\
\hline Neuro QX+ & $5(8 \%)$ & $2(3 \%)$ & $7(11 \%)$ \\
\hline $\mathrm{CT}$ y $\mathrm{CV}++$ & $2(3 \%)$ & $4(7 \%)$ & $6(10 \%)$ \\
\hline Abdominal & $3(5 \%)$ & $2(3 \%)$ & $5(8 \%$ \\
\hline \multicolumn{4}{|l|}{ Comorbilidades } \\
\hline Cardiacas & $6(10 \%)$ & $14(23 \%)$ & $20(33 \%)$ \\
\hline Oncológicas e inmunosupresión & $4(7 \%)$ & $4(7 \%)$ & $8(13 \%)$ \\
\hline Otras & $3(5 \%)$ & $3(5 \%)$ & $6(10 \%)$ \\
\hline \multicolumn{4}{|l|}{ Servicio de procedencia } \\
\hline Pediatría & $4(7 \%)$ & $8(13 \%)$ & $12(20 \%)$ \\
\hline Emergencia & $11(18 \%)$ & $26(43 \%)$ & $37(61 \%)$ \\
\hline Otros & $3(5 \%)$ & $3(5 \%)$ & $6(10 \%)$ \\
\hline \multicolumn{4}{|l|}{ Función neurológica } \\
\hline Normal & $8(13 \%)$ & $25(42 \%)$ & $33(55 \%)$ \\
\hline Discapacidad & $13(22 \%)$ & $14(23 \% 9$ & $27(45 \%)$ \\
\hline
\end{tabular}

*NAV: Neumonía asociada a ventilador; ** CVC: Catéter venoso central; \# UCIP : Unidad de Cuidados Intensivos pediátricos; + NeuroQX: Neuroquirúrgico; ++ CT y CV: Cirugía de tórax y Cardiovascular.

Los principales diagnósticos de ingreso que presentaron los pacientes con NAV se resumen en la Tabla 3. 
Tabla 3. Diagnósticos de Ingreso de los pacientes con neumonía asociada a ventilador (NAV) en la unidad de cuidados intensivos pediátricos del Hospital Rebagliati 2015-2018.

\section{DIAGNÓSTICO}

FRECUENCIA

PORCENTAJE

Choque séptico

12

Neumonía

11

18

Falla cardiaca + malformación cardiaca

9

15

Estado convulsivo

Tos ferina

6

Tumor cerebral

4

3

5

MAV

3

TEC

3

Peritonitis

3

Post paro

3

2

4

60

5

Otros

Total

La Figura 3, muestra la positividad en los cultivos aislados en secreción traqueal con NAV.

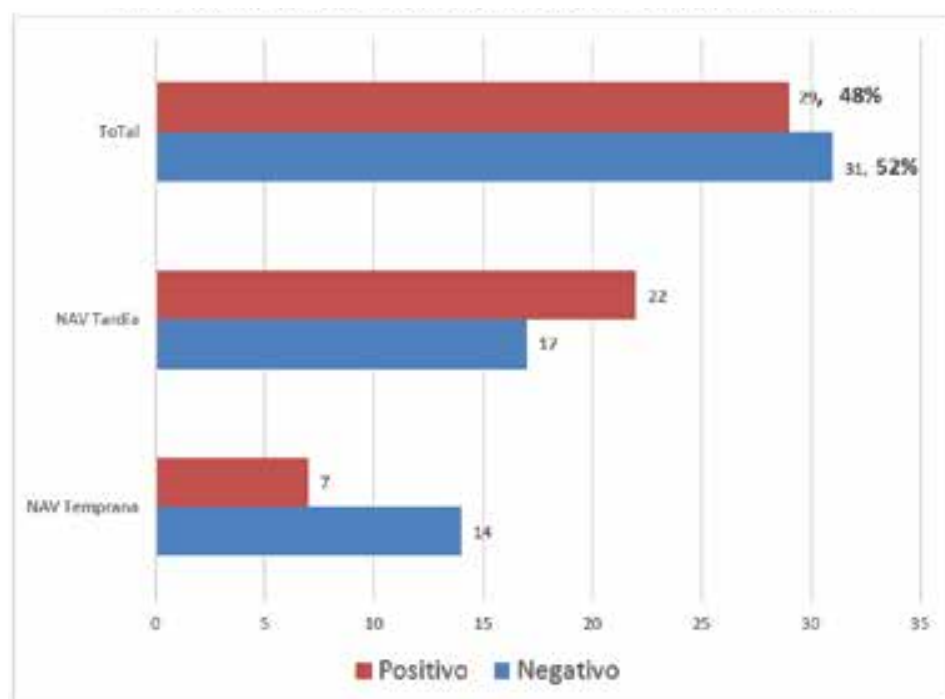

Figura 3. Cultivos positivos de neumonía asociada a ventilador (NAV) en la Unidad de Cuidados Intensivos pediátricos del Hospital Rebagliati 2015-2018.

Los gérmenes aislados en pacientes con NAVy el patrón respectivamente. Fallecieron 9 pacientes de los 60 que de su sensibilidad se presentan en las tablas 4 y 5 se diagnosticaron con NAV equivalente al 15\%.

Tabla 4. Gérmenes aislados en los diagnosticados con neumonía asociada a ventilador (NAV) en la unidad de cuidados intensivos pediátricos del Hospital Rebagliati 2015-2018.

\begin{tabular}{lcccc}
\hline \multicolumn{1}{c}{ GÉRMEN } & NAV TEMPRANA & NAV TARDíA & TOTAL & PORCENTAJE \\
\hline Stenotrophomona maltophilia & 3 & 10 & 13 & $45 \%$ \\
Pseudomona aureginosa & 1 & 5 & 6 & $21 \%$ \\
Klebsiella pneumoniae & 0 & 2 & 2 & $7 \%$ \\
Staphylococcus aureus & 1 & 1 & 2 & $7 \%$ \\
Acinetobacter Iwoffi & 2 & 0 & 2 & $7 \%$ \\
Acinetobacter baumannii & 0 & 1 & 1 & $3 \%$ \\
Enterococcus faecalls & 0 & 1 & 1 & $3 \%$ \\
Escherichia coli & 0 & 1 & 1 & $3 \%$ \\
Serratia marcescens & 0 & 1 & 1 & $3 \%$ \\
Total & 7 & 22 & 29 & $100 \%$ \\
\hline
\end{tabular}


Tabla 5. Patrón de sensibilidad de los gérmenes más frecuentes en neumonía asociada a ventilador (NAV) en la unidad de cuidados intensivos pediátricos del Hospital Rebagliati 2015-2018.

\begin{tabular}{|c|c|c|c|c|c|c|c|c|c|c|c|c|}
\hline Microorganismo & AMX & CTX & CTZ & CEF & CFTX & IMI & MER & CTMX & CIP & PIP/TAZ & COLI & ERT \\
\hline P. AERUGINOSA & $90,5 \%$ & 0 & 0 & $78,9 \%$ & - & $50 \%$ & $62 \%$ & - & $95 \%$ & $0 \%$ & $94,4 \%$ & \\
\hline ACINETOBACTER BAUMANI & $0 \%$ & - & $0 \%$ & $0 \%$ & $0 \%$ & $0 \%$ & $100 \%$ & $0 \%$ & $0 \%$ & - & - & - \\
\hline K. PNEUMONIAE & $100 \%$ & - & $25 \%$ & $25 \%$ & $22 \%$ & $100 \%$ & $100 \%$ & $0 \%$ & $50 \%$ & $100 \%$ & $100 \%$ & $100 \%$ \\
\hline STENOTROPHONA M. & - & - & $63 \%$ & - & - & - & - & $81 \%$ & $100 \%$ & - & - & - \\
\hline E. COLI & $100 \%$ & $0 \%$ & $33 \%$ & $0 \%$ & $33 \%$ & $100 \%$ & $100 \%$ & $33 \%$ & $33 \%$ & $67 \%$ & $100 \%$ & $100 \%$ \\
\hline ACINETOBACTER WOFFI & $100 \%$ & - & $0 \%$ & $0 \%$ & $0 \%$ & & $0 \%$ & $0 \%$ & $0 \%$ & - & - & - \\
\hline
\end{tabular}

AMK: Amikacina; CTX: Ceftriaxona; CTZ: Ceftazidima; CEF: Cefepime; CFTX: Cefotaxima; IMI: Imipenem;

MER: Meropenem; CTMX: Cotrimoxazol; CIP: Ciprofloxacino; PIP/TAZ: Piperacilina Tazobactam ERT: Ertapenem.

\section{DISCUSIÓN}

La causa más frecuente de infección asociada a dispositivo en nuestra UCIP es la neumonía asociada a ventilador (NAV) que en estos 4 años se han mantenido por debajo del estándar de 12 por 1000 días de VM, teniendo incluso en el 2017 una tasa de 5.6. En el Perú la notificación de los establecimientos que reportan infecciones intrahospitalarias desde el año 2005 a la fecha se ha ido incrementando cada año, al igual que lo reportado en nuestra UCIP, la tasa de incidencia más alta dentro de las infecciones asociadas a dispositivos, correspondió a las NAV en cuidados intensivos de adultos $11,67 \times 1000$ días de exposición a ventilación mecánica, mientras que en neonatología fue $5,43 \times 1000$ días de exposición a ventilación mecánica ${ }^{13}$. En España, a través de un estudio Nacional de Vigilancia de Infección Nosocomial proveniente de datos de 26 UCI pediátricas en el año 2014 la tasa de NAV fue de 8,51/1000 días de VM. sin embargo hay publicaciones con tasas mucho menores, 0,9-2,6 episodios por 1000 días VM, esto debido a las medidas preventivas instaladas en tales instituciones sin embargo en algunos trabajos se reportan hasta 61 por 1000 días $\mathrm{VM}^{3,14,12}$.

La clasificación de NAV temprana y tardía permite buscar y evitar algunos factores de riesgo, $y$ en el caso de las NAV tardías, a los gérmenes resistentes que se producen debido al uso de antibióticos previos, presión selectiva, transmisión cruzada y colonización de fuentes ambientales de UCI.
NAV, fueron tardías, a diferencia de otros que refieren que aproximadamente que más de la mitad de todos los episodios de NAV ocurren tempranamente. En otros trabajos la NAV temprana es superior al 70\%: esto podría deberse como vemos en los resultados, a los días de hospitalización y VM en nuestra UCIP, a la complejidad de pacientes que manejamos, las comorbilidades que presentan, cirugías complejas realizadas, pudieran determinar mayor frecuencia de las NAV tardías ${ }^{3,8,15}$.

Existen diferentes factores que favorecen la aparición de la NAV. Estos están relacionados con factores extrínsecos o intrínsecos al huésped. La edad, como factor intríseco es un factor importante para NAV. En nuestros resultados se observa un mayor porcentaje de pacientes menores de 6 meses con episodios de NAV, en total los menores de 3 años constituyen el $68 \%$, y de ellos la NAV tardía es más frecuente, coincidiendo con diversos reportes en los que la edad menor de 1 años es un factor de riesgo importante para NAV, otros refieren que el grupo de edad de 0 a 5 años fue el doble que el del grupo de edad de 5 a 12 años $^{14,15}$.

Otros de los factores intrínsecos es el estado nutricional, la presencia de comorbilidades y función neurológica del paciente. En el presente estudio el $30 \%$ de los pacientes son desnutridos, teniendo un mayor porcentaje de desnutridos las NAV tardía. La obesidad y sobrepeso están presentes en el $13 \%$ de las NAV; el estado nutricional afecta directamente a la respuesta frente a un proceso infeccioso y los hace más susceptibles a padecerlos a 
los pacientes malnutridos (desnutridos y obesos) ${ }^{9}$. En nuestro estudio se registra un $18 \%$ de desnutridos, lo que evidencia que en los pacientes con NAV la desnutrición es mucho mayor. El 56\% de las NAV eran de pacientes con comorbilidades, siendo las más frecuentes las cardíacas con $33 \%$ y el $45 \%$ con algún grado de discapacidad neurológica. El diagnóstico de ingreso más frecuentemente reportado fue el schock séptico, siendo el caso que otros estudios reportan al Síndrome de Distres respiratorio (ARDS) como el más frecuente $\mathrm{e}^{3,8,9,16}$.

Dentro de los factores extrínsecos, múltiples estudios han vinculado una mayor duración de la ventilación mecánica de 5-11 días, y algunos consideran a este el mayor factor de riesgo para NAV, ocurriendo en el $94 \%$ de los casos en la primera semana de VM. Apostolopoulou et al. reporta picos de NAV entre el día 5 y el día 9 de ventilación mecánica ${ }^{15}$. Nosotros reportamos una mediana de los días de VM de 15 días, siendo mayor los días de VM en las neumonías tardías, por ello también se explicaría su mayor frecuencia. El 25\% de pacientes reingresaron a VM, esto asociado a reintubaciones, que es señalado como otro factor de riesgo para $\mathrm{NAV}^{3,8,15,17}$.

Es importante también señalar los otros dispositivos invasivos que tuvieron los pacientes que desarrollaron NAV, en el caso del Catéter venoso central (CVC) tuvo una duración media de 13 días, siendo similar en la NAV temprana y tardía; y los días de sonda urinaria es 9 días en la NAV temprana comparada con 14 días de la tardía.

Una estadía larga en UCIP también es otro factor de riesgo reportado debido a que podría condicionar a tener mayor colonización con gérmenes intrahospitalarios; en el presente estudio el promedio de días de hospitalización de pacientes con NAV fue de más de 30 días en todas las NAV, y en la tardía 38 días, teniendo el rango superior 207 días. Esta estancia podría explicar la frecuencia mayor de NAV tardías: algunos de los reportes muestran mayor riesgo con estancias promedios 20 a 34 días $^{14}$. Los días promedio de los pacientes hospitalizados en nuestra UCIP es de 8 , por lo que la NAV aumenta considerablemente la estancia hospitalaria ${ }^{14}$.

Otros de los factores extrínsecos que se mencionan son las cirugías realizadas. En nuestro estudio el $30 \%$ de las NAV fueron post operados y más frecuentemente las neuroquirúrgicas, debiendo mencionar que el servicio evaluado es una UCIP general y más del $50 \%$ de los pacientes que recibe son post operados. los pacientes postoperatorios tenían neumonía9,17.

El $48 \%$ de los cultivos resultaron positivos, siendo más frecuente la positividad en las NAV tardías. Los gérmenes más frecuentes fueron la Stenotrophomona maltophilia y la Pseudomona auriginosa, siendo el caso que éstas se aislaron principalmente en las NAV tardías. El aislamiento de esta última coincide con la mayoría de estudios en que se reporta como los gérmenes más frecuentes de NAV a los gram negativos, encabezados por la Pseudomona auriginosa, acinetobacter baumani y Klebsiella pneumoniae ${ }^{17}$. Nuestra UCIP reporta trimestralmente sobre los gérmenes de nuestra flora por lo que se tiene el conocimiento del patrón de sensibilidad, siendo la Stenotrophomona maltophilia sensible en $81 \%$ cotrimoxazol y en el caso de la pseudomona auriginosa muestra una sensibilidad casi de $80 \%$ al cefepime y $90 \%$ a amikacina y sólo entre el $50 \%$ a $60 \%$ a los carbapenemens. Este patrón de sensibilidad de pseudomona que es menos sensible a los carbapenems y con mayor sensibilidad a cefepime, se debe a la resistencia que adquiere esta bacteria por el mecanismo de porinas de membrana y mecanismos de penetración. La penetración del imipenem y otros carbapenems a través de la membrana externa de $P$. aeruginosa ocurre a través de la porina OprD, específica para el transporte de aminoácidos dibásicos y de glutamato. Presumiblemente, la analogía estructural entre los carbapenems y los aminoácidos dibásicos explica la capacidad de las primeras para penetrar usando OprD. De acuerdo a estos datos realizamos tratamientos iniciales para NAV. Las recomendaciones es que en las NAV tardías se traten 14 días a más. Los gram positivos son reportados en baja proporción similar a lo reportado en el presente ${ }^{17,18,19,20}$.

\section{CONCLUSIÓN}

La tasa anual promedio de NAV en el servicio evaluado fue 9.8 por 1000 días de VM, concordando con estándares internacionales, siendo el tipo más frecuente la tardía (65\%). La NAV más frecuente en menores de 6 meses y sexo masculino.

La desnutrición, cardiopatía congénita, tiempo de ventilación mecánica, días de hospitalización y cirugías se encontraron en mayor proporción en los pacientes con NAV.

Los gérmenes aislados más frecuentemente fueron Stenotrophomona maltophilia y la Pseudomona auriginosa, con baja proporción de multidrogoresistencia. Finalmente, la mortalidad por NAV fue del $15 \%$. 
Contribuciones de autoría: Los autores participaron en la generación, redacción y aprobación final del artículo original.

Financiamiento: Autofinanciado.

Conflicto de interés: Los autores declaran no tener conflicto de intereses en la publicación de este artículo.
Recibido: 02 de diciembre 2018

Aprobado: 22 de mayo 2019

Correspondencia: Liliana Paola Cieza Yamunaqué

Dirección: Jr. Belisario Flores 238 Dpto 301 - Lince. Lima 14, Lima - Perú

Teléfono: +51-990452547

Correo: liciya04@hotmail.com

\section{REFERENCIAS BIBLIOGRÁFICAS}

1. Fan Y, Gao F, Wu Y, Zhang J, Zhu M, Xiong L. Does ventilator-associated event surveillance detect ventilator-associated pneumonia in intensive care units? A systematic review and meta-analysis. Crit Care [Internet]. 2016 Dec 24;20(1):338. Available from: https://www.ncbi.nlm.nih.gov/ pubmed/27772529

2. CDC. Pneumonia (Ventilator-associated [VAP] and non-ventilatorassociated Pneumonia [PNEU]) EventNo Title. In: National Healthcare Safety Network (NHSN) Patient Safety Component Manual. 2019. p. cap6,1-16.

3. Gerardo Vizmanos Lamotte CM de V. Neumonía adquirida en el hospital. Protoc diagn ter pediatr. 2017;1:147-56.

4. Mourani PM, Sontag MK. Ventilator-Associated Pneumonia in Critically II Children. Pediatr Clin North Am [Internet]. 2017 Oct;64(5):1039-

56. Available from: https://linkinghub.elsevier.com/retrieve/pii/ S0031395517300731

5. Wanda cornistein ${ }^{1}$ Àngel $\mathrm{m}$. colque ${ }^{2}$, María Inés staneloni ${ }^{3} \mathrm{MML}$, Mónica Lares ${ }^{5}$, Ana Laura González ${ }^{6}$ AFG, Carbone ${ }^{8}$ E. Neumonía Asociada a Ventilación Mecánica. Actualización y Recomendaciones Inter-sociedades, Sociedad Argentina de Infectología -Sociedad Argentina de Terapia Intensiva. Medicina (B Aires). 2018;78(2):99106. Available from: https://www. ncbi.nlm.nih.gov/ pubmed/28941534

6. Vijay G, Mandal A, Sankar J, Kapil A, Lodha R, Kabra SK. Ventilator Associated Pneumonia in Pediatric Intensive Care Unit: Incidence Risk Factors and Etiological Agents. Indian J Pediatr [Internet]. 2018 Oct 4;85(10):861-6. Available from: https://www.ncbi.nlm.nih.gov/ pubmed/29616405

7. Mietto C, Pinciroli R, Patel N, Berra L. Ventilator Associated Pneumonia: Evolving Definitions and Preventive Strategies. Respir Care [Internet]. 2013 Jun 1;58(6):990-1007. Available from: https:// www.ncbi.nlm.nih. gov/pubmed/23709196

8. Charles MP. Incidence and risk factors of ventilator associated pneumonia in a tertiary care hospital. Australas Med J [Internet]. 2013 May 1;6(4):178-82. Available from: https://www.ncbi.nlm.nih.gov/ pubmed/23671462

9. Charles MP. Ventilator-associated pneumonia. Australas Med J [Internet]. 2014 Aug 31;334-44. Available from: https://www.ncbi.nlm. nih.gov/pubmed/25279009

10. Ministerio de Salud - Perú. Protocolo: estudio prevalencia de infecciones intrahospitalarias. 2014. 80 p.
10. EsSalud. "'Vigilancia de las infecciones asociadas a la atención de salud en el Seguro Social de Salud- ESSALUD" Directiva N16 -GCPS-ESSALUD-2016. Perú; 2016. 75 p.

11. Nair G, Niederman M. Using Ventilator-Associated Pneumonia Rates as a Health Care Quality Indicator: A Contentious Concept. Semin Respir Crit Care Med [Internet]. 2017 Jun 4;38(03):237-44. Available from: s-0037-1602580

12. Salud $M$ de SDG de $P$ de SD de $C$ en. Documento técnico: Lineamientos para la vigilancia, prevención y control de las infecciones asociadas a la atención de salud. http://bvs. Perú; 2016. 1-30 p.

13. Venkatachalam V, Hendley JO, Willson DF. The diagnostic dilemma of ventilator-associated pneumonia in critically ill children*. Pediatr Crit Care Med [Internet]. 2011 May;12(3):286-96. Available from: https:// www.ncbi.nlm.nih.gov/pubmed/21037503

14. Barbier F, Andremont A, Wolff M, Bouadma L. Hospital-acquired pneumonia and ventilator-associated pneumonia. Curr Opin Pulm Med [Internet]. 2013 May;19(3):216-28. Available from: https://www.ncbi. nlm.nih.gov/pubmed/23524477

15. Cieza L. Soporte nutricional en el paciente pediátrico crítico. Rev la Fac Med Humana [Internet]. 2018 Oct 8;18(4). Available from: http://revistas.urp.edu.pe/index.php/RFMH/article/view/1737

16. Afanador CE, Donado JH CC. Factores de riesgo para neumonía asociada al ventilador en un hospital de tercer nivel de la ciudad de Medellín: estudio de casos y controles. Med UPB. 2008;27(1):25-32.

17. Diaz E, Lorente L, Valles J, Rello J. Neumonía asociada a la ventilación mecánica. Med Intensiva [Internet]. 2010 Jun;34(5):31824. Available from: https://linkinghub.elsevier.com/retrieve/pii/ S0210569110000896

18. Iosifidis E, Stabouli S, Tsolaki A, Sigounas V, Panagiotidou E-B, Sdougka $M$, et al. Diagnosing ventilator-associated pneumonia in pediatric intensive care. Am J Infect Control [Internet]. 2015 Apr;43(4):390-3. Available from: https://www.ncbi.nlm.nih.gov/ pubmed/25704257

19. Liliana Cieza Yumunaqué ECP, Manrique 1 Manuel Reyes. Perfi microbiológico de la Unidad de Cuidados Intensivos Pediátricos del Hospital Rebagliati 2014-2016.Rev Médica Rebagliati. 2018;1(2):18-25. 